\title{
Computational Intelligence-based Semantic Image Background Identification using Colour-Texture Feature
}

\author{
Mohammad Al-Azawi \\ Oman College of Management \& Technology \\ Barka, Oman
}

\begin{abstract}
Most of the studies in image contents recognition and identification focus on identifying the objects that the image contains. Although, background identification is an important part of any image contents identification and description, it has received less attention. Background identification is as important as the objects in the image in describing the image contents. This paper presents a new algorithm to identify the background in an image which utilises the principles of human image understanding and the way they describe the contents of the image. Because backgrounds are with less variation as compared to objects variations in different images, neural network can be used in recognising them. Usually backgrounds can be described based on their texture and colour; thus, these two features will be used in the identification process. Texture is described using measures extracted from Grey Level Co-occurrence Matrices (GLCMs and singular GLCM). Since different textures may produce similar GLCMs, colour features can be used to discriminate among them. Fuzzy Standard Colours are presented in this research which is a simulation to the way the human describe the colours. Additionally, Fuzzy intelligence is used to combine the two features to decide the type of the background.
\end{abstract}

\section{General Terms}

Image Processing, Computational Intelligence, Neural Network, Fuzzy Logic, Image Contents Identification, Image understanding, Fuzzy Standard Colours.

\section{Keywords}

Image, Fuzzy, Background, Identification.

\section{INTRODUCTION}

Image contents recognition and identification is still a challenging topic for researchers. Although, numerous researches are published every year, still the obtained results are not quite satisfactory. Studying the principles of Human Vision System (HVS) has inspired many researchers to design images identification systems that utilise these principles.

In order to give the machine the ability to recognise and understand the contents of the image, one needs to convert the image from the visual, or spatial domain, into a form that can be processed and measured by the machine which is the features domain. After this conversion, the features in the features domain are converted into descriptors in the description domain. Features domain includes many types of features such as colour and texture which are widely used to describe the contents of the image.

Texture feature has been employed widely in image identification since colour is not sufficient to describe them. Several measures have been proposed to describe the texture such as wavelet, Gabor filters and GLCMs. GLCMs provide description for the relation of the pixel with the surrounding pixels, which can be considered as a description of the texture. Since GLCMs are extracted from the grey level image, which means, only the intensity values are used in determining GLCMs, then the colour should be used as well to give a complete description to the background.

Computational Intelligence (CI) techniques, such as Neural Networks (NNs and singular NN) and Fuzzy Intelligence (FI) are used to mimic the human abilities in identifying the images contents. Various NNs have been adopted due to their ability of learning and being used in the recognition phase. Since both the inputs and the corresponding outputs can be provided to the NNs, then supervised learning can be used and it works efficiently. Different learning techniques such as Back Propagation (BP), Evolutionary Learning (EL) and Delta Rule (DR) can be used for training the NNs. Fuzzy Intelligence is used to combine the properties that are obtained from NN's and to specify the type of the background.

Numerous literatures have been published in the field of image content recognition, texture analysis, and computational intelligence. Most of the proposed algorithms are domain-oriented; i.e. they are not general and cannot be applied on general image datasets. In other words, they deal with a specific field of applications such as medical, GSI, weather, geological applications, etc. Medical applications such as tumour identification and MRI recognition were the focus of numerous researches such as Ref. [1], [2], [3] and many others. Atmosphere and weather applications are another fields that utilise GLCMs and NNs in their identification process [4] [5]. Similar techniques have been used in applications such as in fabric defection identification [6], coal/rock identification [7], fruit identification [8] and others.

In all of the aforementioned applications, in additional to other application, it is noticed that the images used in the dataset are related to each other, e.g. skin tissues, MRI, clouds, rocks, etc. In each images dataset, the images contain very similar texture and usually the algorithm role is to identify the abnormal cases like in tumour identification. NNs work effectively in such applications since they do not need to be retrained frequently when the data is changed.

In this research, GLCMs, NNs and Fuzzy intelligence, in additional to the colour features, are used to design a new algorithm that can identify different backgrounds in an image. The proposed technique overcomes the problem of retrain the NNs frequently when a new texture is added, especially when it is applied on images from different domains. Instead of retraining the NNs frequently, Fuzzy Interface System (FIS) is used, which made the proposed algorithm more flexible by 
defining new backgrounds in the rulebase based on the results obtained from the NNs.

\section{BACKGROUND AND THEORY}

This section focuses on the background and the needed theory upon which the rest of the paper will be built. Image background identification is an important part of image contents identification as it gives information about the nature of the image. Most of the image contents identification algorithms focus on the objects in the image without giving sufficient information about the background. When a person describes a scene, he, usually, gives description for the object and the surrounding environment such as a ball in a field or playing yard. Based on this, background identification is as important as the object identification.

Colour feature is used to describe the contents and the nature of the image in many applications. The most well-known representation for colour feature is the histogram, which gives good description for colour distribution in the image but it suffers from a certain weakness, which is that it losses the space information of the colour [9]. In addition, different image may have similar histograms such as the sky and the sea or tree leaves and grass; therefore, texture is used to solve such problems. When it refers to the description of the image's texture, texture's statistical feature and structural feature, as well as, frequency domain (spectral) features can be used [10]. Although texture is not well defined, as in the case of colour feature, still it can give good description for the contents in images that contain objects such as cloud, trees, bricks, and fabric. Texture features can be extracted using various approaches such as Gabor filter, wavelet transform and local statistics measures. The paper's main interest is in the statistical approach which includes many techniques such as the Moments of Intensity and Grey Level Co-occurrence Matrices.

\subsection{Grey Level Co-occurrence Matrices (GLCMs)}

GLCMs, which were presented by Haralick [11], are used to describe the relation of each pixel's grey intensity level with its neighbours. They are based on the joint probability distributions of pairs of pixels, each GLCM can be specified in a matrix of relative frequencies with which two neighbouring pixels separated by a distance $\delta$ and angle $\theta$ occur on the image, one with grey level $i$ and the other with grey level $j[12]$.

The relative frequencies of grey level pairs of pixels separated by a distance $\delta$ in the direction $\theta$ are combined to form a relative displacement vector $D=(\delta, \theta)$. This vector is computed and stored in the GLCMs $(G)$. These matrices are used to extract second-order statistical texture features. GLCMs are used to describe the texture since they measure the relation between the adjacent pixels displaced by a certain distance. GLCMs are, usually, described in terms of displacement $\delta$ and angle $\theta$, i.e. $G L C M=f(x, y, \delta, \theta)$. The angle $\theta$ often takes standard values such as, $0^{\circ}, 90^{\circ}, 180^{\circ}$, etc. Instead of using the angle $\theta$, two different displacements $\delta_{x}$ and $\delta_{y}$ can be used. The GLCM is then can be defined as follows:

$G(a, b)=\frac{1}{\left(H-\delta_{y}\right)\left(W-\delta_{x}\right)} \sum_{i=0}^{H-\delta_{y}} \sum_{j=0}^{W-\delta_{x}} g(a, b)$ $g(a, b)= \begin{cases}1 & \text { if } I(x, y)=a \wedge I\left(x+\delta_{x}, y+\delta_{y}\right)=b \\ 0 \quad \text { otherwise }\end{cases}$

where $G$ is a GLCM with size of $N \times N$, and $N$ is the number of grey levels (intensity levels) in the image.

Several measures can be extracted from the GLCMs which can be used to describe the texture. In our application, two important features for the texture will be defined; these features are coarseness and regularity.

\subsubsection{Coarseness}

It was noticed that, the shape of the GLCMs is dependent upon the coarseness of the texture; this is because in case of fine texture, GLCMs extraction process produces GLCMs with a clear spot. For instance, assume that $G(x, y, \delta, 0)$ is calculated, which means only the horizontal relationship between the adjacent pixels is considered. For coarse texture, if the width of the textures is $w$, then if $\delta$ is less than $w$, the value of $g$ at some grey level $a$ with respect to itself will be higher, i.e. $\mathrm{g}(a, a)$ will be higher than the case of fine texture in which the ratio of $\delta / w$ is higher than that for coarse texture. In other words, the number of pixels at which $I(x, y)=I\left(x+\delta_{x}, y+\delta_{y}\right)$ is higher in coarse texture than in fine texture. Thus, the width of the GLCMs shall be considered as a measure of the coarseness of the texture.

From Table 1, it is clear that for fine texture, the GLCM is concentrated in one region (spot) for fine texture, while its size is larger and there are some values corresponding to the same intensity values for coarse texture. The location of the spot in the 2D GLCM or the centre in 1D GLCM has been affected by the brightness of the image, which may affect the result slightly. This problem can be solved by shifting the centre of the spot to a fixed position.

Table 1. The effect of coarseness on GLCM

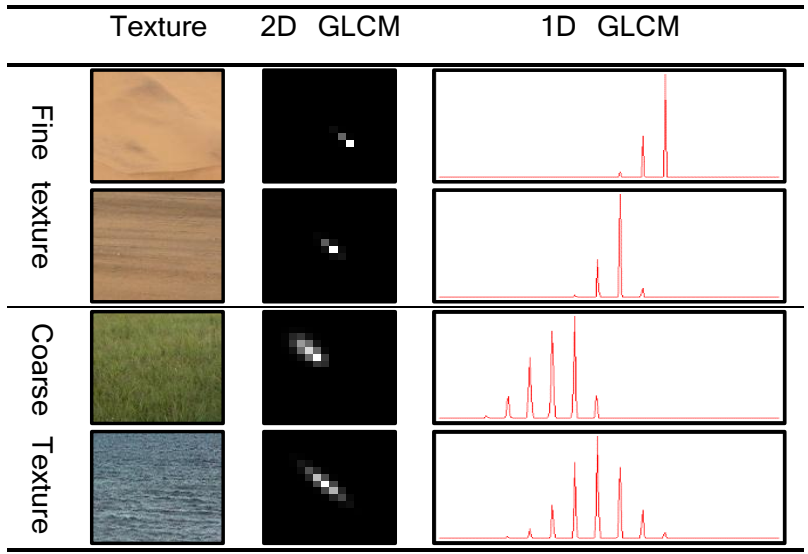

\subsubsection{Regularity}

Regularity is another important property of the texture that can affect the shape of the GLCM. Regularity measures the repetition of the texture in the image, e.g. the grass is regular since the same texture is repeated continuously, while the cloudy sky is irregular. The irregularity effect on the GLCMs is characterised by having more than one spot in the GLCMs, one spot for each part, e.g. in the case of cloudy sky there are two spots one for the blue part and one for the clouds. Thus, the GLCMs shall be divided into more than one part i.e. $\mathrm{G}=\mathrm{G}_{1}+\mathrm{G}_{2}+\cdots+\mathrm{G}_{\mathrm{m}}$; where $G_{i}$ for $i=1,2, \ldots m$ are the GLCMs corresponding to different parts of the texture, and each one produces its own spot since they are calculated for a 
specific range of grey levels. Figure 1 shows the effect of irregularity on GLCMs; from the figure, it is obvious that there are two major peaks for the 1D GLCMs: one for the clouds and one for the blue background and two spots in the 2D GLCM for the same reason.

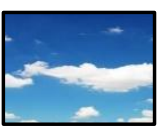

(a)

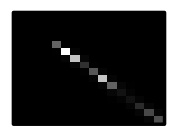

(b)

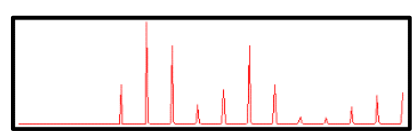

(c)
Fig 1: The effect of irregularity on the GLCM, (a) original image, (b) 2D GLCM 16 grey levels, (c) 1D GLCM 16 grey levels

To measure the texture features we shall use different measures that can be extracted from 1D GLCMs, such as the mean $\mu$, standard deviation $\sigma$, range $\rho$, number of peaks (tops) $\tau$, and average of the tops $\alpha$. Each one of the measures is affected by the properties of the texture, e.g. the coarseness affects the range, and the regularity increases the number of peaks.

\subsection{Neural Networks}

Neural Computing is widely used in image identification algorithms, since NNs have the ability of adaptation and learning. In such algorithms, the input to the $\mathrm{NN}$ is a vector of features that are extracted from images. A set of input vectors is used to train the $\mathrm{NN}$ so it can recognise other vectors that are extracted from other images. Although both supervised and unsupervised learning techniques can be used in image classification, in most of the proposed systems, supervised learning is used because it gives the possibility to improve the identification performance. Such retrieval systems utilise user feedback as one of the inputs to the supervised learning NN.

NNs suffer from a certain constraint, which is they can be used effectively to identify images that are related to each other, such as MRI, therefore, they are widely used in medical applications; but it is not feasible to use them in identifying image contents with different natures since they need to be retrained with every new type. Thus, most of the applications of NNs in image retrieval systems are in relevance feedback [13], general classification [14] and human computer interaction [15].

\subsection{Fuzzy Intelligence}

Fuzzy intelligence provides excellent way to convert quantitative numerical values into qualitative values by using linguistic variables, which can store linguistic values instead of numerical values. These linguistic values are specified by the selected membership function. To process the linguistic variables, we need to use what is called Fuzzy rules, which is a set of (if-then) relationships. The set of linguistic variables is known as the database and the set of rules is known as rulebase, these two together form important part of the Fuzzy interface system FIS. The use of Fuzzy intelligence in the proposed algorithm is to simulate the uncertainty in the human visual system.

\section{PROPOSED ALGORITHM}

The proposed algorithm has been built upon the following assumptions: firstly, no frequent retraining for the NNs should be performed; secondly, identifying the texture coarseness and regularity in additional to the colour using NNs; and thirdly, using Fuzzy intelligence to combine the results obtained from the NNs to decide the texture type. The role of the NNs in the proposed algorithm is to identify the background properties such as coarseness, regularity and colour.

A bank of NNs, which consists of three NNs, was used. The purpose of the first $\mathrm{NN}$ is to identify the coarseness, the second one is used to identify the regularity and the last one is dedicated to identifying the colour. The inputs to the first two NNs are the measures of the reduced 1D GLCMs. The idea of using the reduced GLCMs and not the original ones is to reduce the complexity of calculating the measures of the input vectors. The reduction in GLCM does not affect the result much since the GLCMs maintain the correspondence with the texture. Fig 2 shows the effect of reducing the size of GLCMs from 256 grey level (GL) to $16 \mathrm{GL}$.

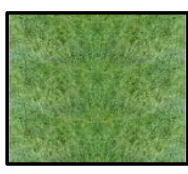

(a)

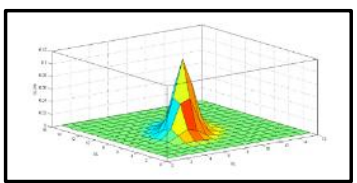

(d)

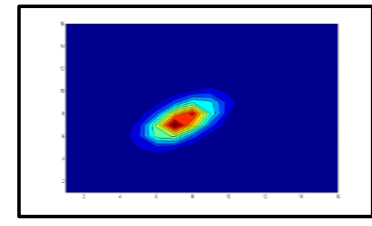

(f)

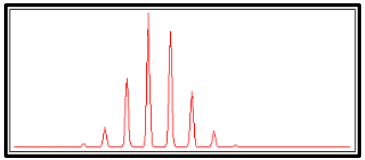

(h)

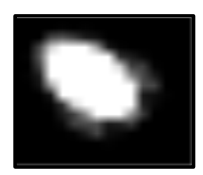

(b)

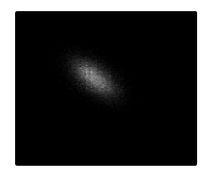

(c)

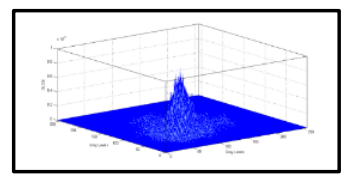

(e)

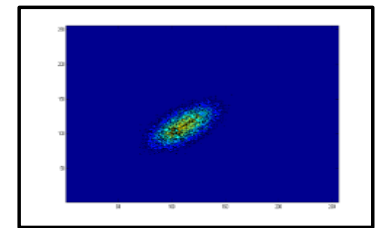

(g)

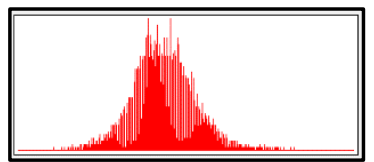

(i)

Fig 2: 16 and 256 GL GLCM (a) original image, (b) 16 GL GLCM, (c) 256 GL GLCM, (d) 16 GL 2D GLCM histogram, (e) 256 GL 2D GLCM histogram, (f) 16 GL 3D GLCM contour, (g) 16 GL 3D GLCM contour, (h) 16 GL 1D GLCM histogram, (i) 256 GL 1D GLCM histogram

The input to the third NN is the colour components of the image. Hue, Saturation and Value (HSV) colour system is used in identifying the colour since it is analogy to Human Vision Systems and Munsell Well. It is possible to obtain most of the Fuzzy Standard Colours (FSC) using HSV. In FSC, we aim at finding a way to describe the colour in a way similar to human description, which means, to give a possessive values for the colour, which might be represented by a Fuzzy membership function. For example, we may describe the colour as Reddish Brown, which means the value for the brown membership value is higher than that for red, but still there is a value for the red.

Figure 3 shows the block diagram of the proposed algorithm. NN1 is used to identify the coarseness, NN2 is used to identify the regularity and NN3 is used to identify the colours.

The FIS consists of the linguistic database which contains linguistic variable such as Colour $=\{$ Green, Brown, Blue, etc. $\}$, Coarseness $=\{$ very fine, fine, course, very Coarse $\}$, Regularity $=\{$ very regular, regular, irregular, very regular $\}$ 
and finally texture $=\{$ field grass, beach sand, desert, sky, cloudy sky, etc. $\}$. In additional to the linguistic database, rulebase is defined as well. Rules such as "IF the coarseness IS smooth and the Regularity IS very regular and the Colour IS light brown THEN the texture IS Desert Sand" can be used to identify the texture.

\section{EXPERIMENTAL RESULTS}

The above algorithm has been applied on different sets of backgrounds with different textures. The standard colours used to train the neural network were obtained from different users feedbacks with different backgrounds. The standard colours set contained colours like red, brown, green, blue, yellow, white, black, purple, orange, and grey.

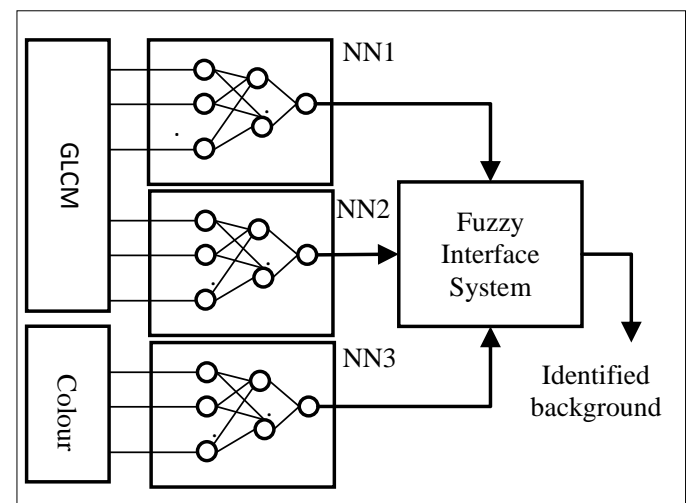

Fig 3: The proposed algorithm block diagram

The colour experimental results are shown in Figure 4, in which different colours have been labelled based on the Hue value with constant value for the saturation and value.

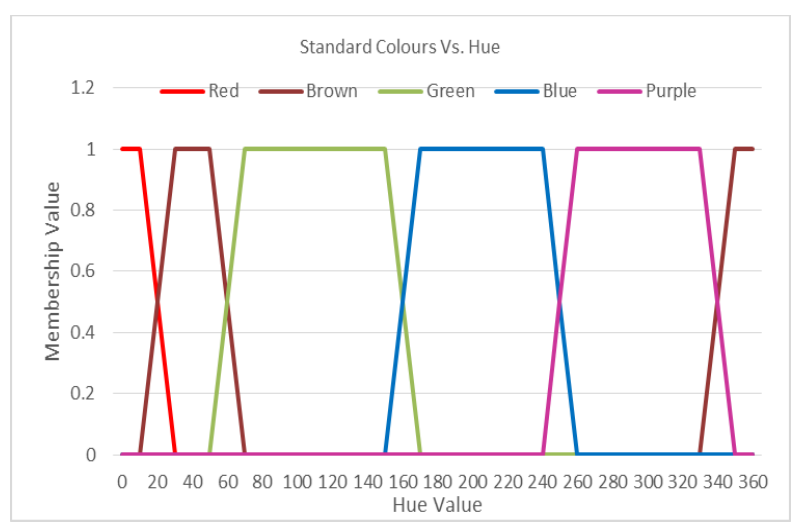

Fig 4: FSC vs. Hue, Saturation $=0.5$ and Value $=0.5$

Some other FSC can be obtained by changing the values of the Saturation and value. The names of the FSC along with the HSV values are used to train the neural network NN3, and then it is used to identify other colours. NN3 has number of outputs equals to the number of FSC used. Thus, the output corresponding to a specific colour is considered as the membership value for that colour, e.g. if the output of the red colour is 0.7 and that of the brown is 0.3 , that means the colour is brownish red.

In the same way, the output of NN1 gives how coarse the texture is, e.g. if the output is 0.9 means very coarse, 0.7 means coarse, and 0.2 means fine. Finally, NN2 gives how regular the texture is. The outputs of the neural networks are then used to define the type of the texture in FIS. The following table shows examples of the results obtained from applying the proposed algorithm.
From Table 2 one can notice that in case 1 , $\boldsymbol{\sigma}$ is smaller than that of case 2, which means that the first textures is with higher coarseness than the second one. The value of $\boldsymbol{\rho}$ in (1) is higher than that in (2) for the same reason. The number of peaks in (1) is 1 while in (2) is 3 which means that the first image is regular and the second one is with less regularity.

Table 2. Measures obtained from applying the proposed algorithm

\begin{tabular}{|c|c|c|c|c|c|c|c|}
\hline \# & Image & $\boldsymbol{\mu}$ & $\sigma$ & $\rho$ & $\boldsymbol{\tau}$ & $\propto$ & Colour \\
\hline 1 & & 104 & 23 & 151 & 1 & 1 & $\begin{array}{c}0.6: \\
\text { Green } \\
0.1: \\
\text { Brown }\end{array}$ \\
\hline 2 & & 137 & 56 & 187 & 3 & 0.64 & $\begin{array}{c}0.9: \\
\text { Blue } \\
0.002: \\
\text { Green }\end{array}$ \\
\hline
\end{tabular}

By using the trained neural networks and the FIS, the results given in Table 3 have been obtained.

Table 3. Results obtained from applying the proposed algorithm

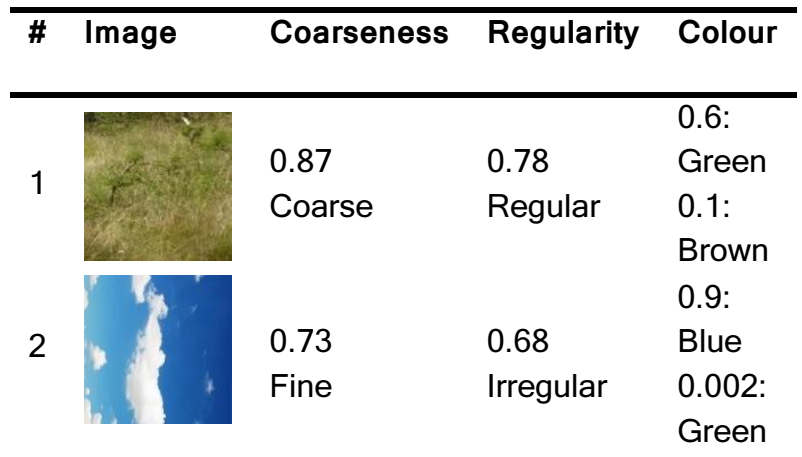

Based on the results in the above table, the description of the image in (1) will be a medium coarseness, pale green grass, and the image in (2) is Day, blue, partially cloudy sky.

Table 4 gives the details of NNs and the training data.

Table 4. Neural Networks details

\begin{tabular}{|c|c|c|c|c|c|c|c|}
\hline \multirow[b]{2}{*}{$\mathrm{NN}$} & \multicolumn{3}{|c|}{$\begin{array}{c}\text { Number of Neurons } \\
\text { in }\end{array}$} & \multirow{2}{*}{ 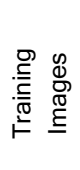 } & \multirow{2}{*}{$\begin{array}{l}\text { n } \\
\frac{\mathcal{D}}{0} \\
\text { யி }\end{array}$} & \multirow{2}{*}{ 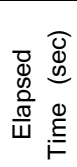 } & \multirow{2}{*}{ 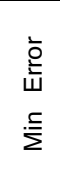 } \\
\hline & 蒙 & $\begin{array}{l}\frac{}{0} \\
\frac{0}{0} \\
\frac{0}{I}\end{array}$ & 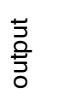 & & & & \\
\hline NN1 & 5 & 5 & 1 & 20 & 1300 & 0.2 & $\begin{array}{c}0.09 \\
9\end{array}$ \\
\hline NN2 & 5 & 5 & 1 & 20 & 25000 & 2.3 & $\begin{array}{c}0.09 \\
9\end{array}$ \\
\hline NN3 & 3 & 3 & 10 & 20 & 11300 & 2.5 & $\begin{array}{c}0.09 \\
9\end{array}$ \\
\hline
\end{tabular}

The number of input neurons for each neural network is equal to the number of measures used in identifying the image properties. The algorithm was tested on 250 different background images and the results are shown in Table 5 . 
Table 5. The percentage of correct results

\begin{tabular}{|c|c|c|c|}
\hline Colour & Regularity & Coarseness & Class \\
\hline $88 \%$ & $84 \%$ & $73 \%$ & $77 \%$ \\
\hline
\end{tabular}

It is noticed from the obtained results that the algorithm worked perfectly on backgrounds with textures such as grass, sand, tree leaves, sea, clouds, stones, and many others. The percentage of wrongly classified backgrounds were in fine clouds and in bricks. In the first case the fine clouds were similar to sea and in the case of bricks, the texture of the brick itself has been considered and not the texture of the wall

Another important merit of the proposed algorithm is that, due to the use of Fuzzy intelligence, it is possible to have more than one class for the same image, for example the image shown in Table 6.

Table 6. A texture may belong to more than one class

\begin{tabular}{|l|l|l|l|l|}
\hline Image & Colour & $\begin{array}{l}\text { Coars } \\
\text { eness }\end{array}$ & $\begin{array}{l}\text { Reg } \\
\text { ulari } \\
\text { ty }\end{array}$ & Class \\
\hline & $\begin{array}{l}0.9999 \\
6 \\
\text { Brown }\end{array}$ & 0.12 & 0.9 & Sand \\
\hline & $\begin{array}{l}0.99 \\
\text { Brown }\end{array}$ & $\begin{array}{l}0.981 \\
0.9933\end{array}$ & 0.76 & Gravel \\
\hline & $\begin{array}{l}0.44 \\
\text { Brown }\end{array}$ & 0.78 & $\begin{array}{l}0.4 \text { Coarse } \\
\text { Sand OR 0.6 } \\
\text { Fine Gravel }\end{array}$ \\
\hline
\end{tabular}

\section{CONCLUSION}

In this paper, an integrated identification system has been proposed. This system can be used to identify the texture image in general, and as an application on the proposed algorithm, we applied it on background identification. The system has some semantic properties since it utilises CI and FIS and inspired by the human visual system. The system uses two main features, which are the colour and texture; these features are used by HVS to identify the contents of the image. Uncertainty is one of the important features of HVS, which can be utilised to have more possibilities or definition of the image. The uncertainty has been represented by using the FIS in terms of colour, coarseness, and regularity membership function.

In addition, NNs play important role in the identification process as they give the possessive value of a specific input to some class. These values can be used, in their turn, to identify the texture properties. The second important advantage of the proposed algorithm is that it overcomes the main limitation of NNs in identification, which is retraining them whenever there is a new class. This limitation has been overcome by using the FIS. Instead of retrain the NN, one can define a new rule in the FIS

The algorithm can be used in many applications which uses texture-colour features in identifying the contents, such as medical, atmosphere, geological and industrial applications.

\section{REFERENCES}

[1] S. Maniar, and J. S. Shah, "Classification of Content Based Medical Image Retrieval Using Texture and Shape
Feature with Neural Network," Int. J. Adv. Appl. Sci., vol. 6, no. 3, pp. 378-384, 2017.

[2] S. Anand, "Segmentation coupled textural feature classification for lung tumor prediction," in IEEE International Conference on Communication Control and Computing Technologies (ICCCCT), 2010.

[3] D. Kadam, S. Gade, M. Uplane and R. Prasad, "An Artificial Neural Network Approach for Brain Tumor Detection Based on Characteristics of GLCM Texture Features,” Int. J. Innov. Eng. Technol., vol. 2, no. 1, pp. 193-199, 2013.

[4] B. Tian, et al., "Neural network-based cloud classification on satellite imagery using textural features," in International Conference on Image Processing, 1997.

[5] B. Tian, et al., "A study of cloud classification with neural networks using spectral and textural features," IEEE Trans. Neural Networks, vol. 10, no. 1, pp. 138$151,1999$.

[6] J. Raheja, et al., "Fabric defect detection based on GLCM and Gabor filter: A comparison," Opt. - Int. J. Light Electron Opt., vol. 124, no. 23, pp. 6469-6474, 2013.

[7] L. Haonan, et al., "Research on identification of coal and waste rock based on GLCM and BP neural network," in 2nd International Conference on Signal Processing Systems (ICSPS), 2010

[8] G. Capizzi, et al., "A novel neural networks-based texture image processing algorithm for orange defects classification,” Int. J. Comput. Sci. Appl., vol. 13, no. 2, pp. 45-60, 2016.

[9] H. Yang, and Z, Xuemei, "Research of Content Based Image Retrieval Technology," in Proceedings of the Third International Symposium on Electronic Commerce and Security Workshops(ISECS '10), 2010.

[10] N. Singhai, and K. Shishir, "A Survey On: Content Based Image Retrieval Systems," Int. J. Comput. Appl., vol. 4, no. 2, pp. 22-26, 2010.

[11] L. Haralick, and R. Watson, "A facet model for image data," Comput. Vision, Graph. Image Process, no. 15, pp. 113-129, 1981.

[12] S. Elberink, and M. Hans-Gerd, "The use of Anisotropic height texture measures for the segmentation of airborne laser scanner data," IAPRS, vol. XXXIII, 2000.

[13] S. Nematipour, J. Shanbehzadeh, and R. Moghadam, "Relevance Feedback Optimization in Content Based Image Retrieval Via Enhanced Radial Basis Function Network," in Proceeding of the International Multiconference of Engineers and Computer Scientists IMECS, 2011.

[14] N. Qazi, and B. Wong, "Semantic based image retrieval through combined classifiers of deep neural network and wavelet decomposition of image signal," in 9th IEEE EUROSIM Congress on Modelling and Simulation (EUROSIM), 2016.

[15] H. Lee, and S. Yoo, "Intelligent image retrieval using neural network," IEICE Trans. Inf. Syst., vol. E84-D, no. 12, pp. 1810-1819, 2001. 